\title{
HERRAMIENTAS EDUCATIVAS EN INTERNET
}

\section{EDUCATIVE TOOLS IN INTERNET}

\section{AUTOR}

José Francisco Durán Medina.

UCLM (Universidad de Castilla La Mancha). Facultad de Educación. Departamento de Pedagogía. Toledo (España).

JoseFrancisco.Duran@uclm.es / JoseFrancisco.Duran@gmail.com

\section{RESUMEN}

En este artículo se realiza una búsqueda documental sobre algunas herramientas educativas en Internet a través del análisis de varios textos que reflejan con claridad el estado o situación del tema a tratar, partiendo de una visión general del lugar que ocupa Internet en la educación. Posteriormente, se analizan varios ejemplos de recursos didácticos concretos utilizados en Internet con fines educativos.

\section{PALABRAS CLAVE}

Educación - Recurso didáctico - Evaluación - Herramienta educativa - Blog - Foro.

\section{ABSTRACT}

In this article a documentary search is realised about some educative tools in Internet through analysis of several texts that reflect with clarity the state or situation of the subject to try, starting since a general vision of the place that occupies Internet in the education. Later, several examples of used concrete tools in Internet with educative aims are analyzed. 


\section{KEY WORDS}

Education - Didactic resource - Evaluation - Educative Tool - Blog - Forum.

\section{ÍNDICE}

1. Fundamentación teórica.

1.1. Marqués, P. (1999): "Concepciones sobre el aprendizaje".

1.2. Santacruz-Valencia, L.P., Aedo, I. y Delgado-Kloos, C. (2004).

1.3. García Aretio, L. (2004): "¿Buscar la información o reproducirla?".

1.4. Hsu, S.; Marques, O.; Hamza, M.K. y Alhalata, B. (1999): "How to design a Virtual Classroom: 10 Easy Steps to Follow".

1.5. Nielsen, J. et al (2001): "Make it usuable".

1.6. Farías, M. y Ponce, R.M. (2004): "Diseño de páginas web para ambientes virtuales de aprendizaje".

1.7.

2. Análisis de ejemplos de herramientas educativas en Internet.

2.1. Blog.

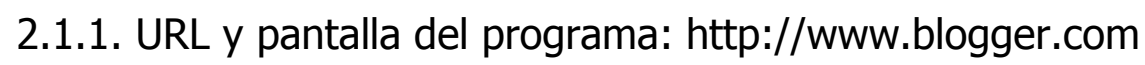

2.1.2. URL y pantalla de ejemplo: http://paginas-webeducativas.blogspot.com

2.1.3. Ventajas educativas.

2.2. Foro.

2.1.1. URL y pantalla del programa: http://www.superforos.com

2.1.2. URL y pantalla de ejemplo: http://nntt.superforos.com

2.1.3. Ventajas educativas.

3. Conclusiones.

4. Bibliografía. 
REVISTA DE LA SEECI.

Durán Medina, José Francisco (2005): Herramientas educativas en Internet. N012. Noviembre. Año IX. Páginas: 84-107

ISSN: 1576-3420 DOI: http://dx.doi.org/10.15198/seeci.2005.12.84-107

5. Webgrafía. 


\section{Fundamentación Teórica.}

Hace ya más de diez años, los pioneros en el uso de las redes telemáticas en la escuela en nuestro país, decían que la comunicación electrónica, la apertura de la escuela al exterior gracias al correo electrónico y a las redes de telecomunicaciones existentes, representa una auténtica revolución para una escuela que hasta hace poco se encontraba cerrada dentro de las cuatro paredes y con muy poca comunicación hacia fuera.

Jordi Adell en el número 11 de la revista Net Conexión en septiembre de 1996, también comentaba que:

"Hoy disponemos de la infraestructura y de los conocimientos necesarios para hacer efectivas algunas de las promesas que la telemática ha realizado al mundo educativo. Si la escuela (léase "las autoridades educativas") da la espalda a las nuevas tecnologías de la información y en particular a la revolución de las comunicaciones digitales, ino estarán dando también la espalda al futuro?" (Adell, 1996).

Para hacer una fundamentación teórica más completa realizaremos una búsqueda documental tras la cual haremos un análisis de algunos textos que reflejen mejor el estado o situación del tema que nos ocupa, partiendo de una visión general del lugar que ocupa Internet en la educación, para ir cerrando poco a poco el círculo y centrarnos más en algunas herramientas concretas, como el Blog o el Foro:

\subsection{Marqués, P. (1999): "Concepciones sobre el aprendizaje".}

El texto comienza con una frase original que anima al lector y le orienta sobre el tema que se va a tratar: "Todos los estudiantes pueden aprender, aunque algunos 
tardan más". Tras esta frase, el lector se planteará las razones causantes de ello y se sentirá motivado para continuar su lectura.

No tarda mucho el autor en despejar nuestras posibles dudas al respecto y señala, con gran acierto a nuestro entender, los tres factores básicos necesarios para todo aprendizaje: poder aprender (inteligencia y otras capacidades y conocimientos previos), saber aprender (experiencia) y querer aprender (motivación). Esta parte del texto, si bien se sitúa al principio del mismo, podríamos decir que realiza una función sintética y actúa como conclusión o resumen de todo lo escrito posteriormente.

El resto del documento, pecando de excesivamente teórico, comenta las operaciones mentales que se realizan en los procesos de aprendizaje (receptivas, retentivas, reflexivas, creativas, expresivas simbólicas, expresivas prácticas), nos refiere la tipología de las actividades de aprendizaje (memorísticas reproductivas, comprensivas), terminando con un breve repaso a las distintas concepciones sobre los procesos de aprendizaje y sus leyes y/o propuestas (perspectiva conductista, teoría del procesamiento de la información, aprendizaje por descubrimiento, aprendizaje significativo, psicología cognitivista, constructivismo, socioconstructivismo).

Aunque como ya hemos señalado la lectura del documento puede resultar poco amena, siempre es positivo un fugaz recordatorio acerca de las teorías más importantes sobre los procesos de aprendizaje, tan esenciales para la docencia como comúnmente olvidadas por los profesionales encargados de impartirla.

\subsection{Santacruz-Valencia, L.P., Aedo, I. y Delgado-Kloos, C. (2004).}

En esta ocasión, teniendo en cuenta que se trata de un trabajo que forma parte de las actividades de investigación financiada por MCYT (Ministerio de Ciencia y Tecnología), y que de sus tres autores, una trabaja en el Departamento de 
Ingeniería Telemática (Liliana Patricia Santacruz-Valencia) y otro en el Departamento de Informática (Ignacio Aedo), no es de extrañar la extraordinaria dificultad que ofrece su lectura y entendimiento.

No obstante, y tras varias lecturas detenidas, podemos extraer algunas ideas principales de este escrito:

- Una de las actividades más recientes en los desarrollos orientados a la web es la web semántica.

- La finalidad de lo que se entiende por web semántica es dotar de significado a todas las clases de información sobre la web.

- Un subconjunto importante de esa información lo representan los objetos de aprendizaje.

- Los objetos de aprendizaje son recursos digitales que se pueden reutilizar en diferentes contextos para lograr un objetivo de aprendizaje particular.

Todos estos contenidos se pueden encontrar en la primera parte del documento titulada "Resumen" o "Summary". Después de ello, el texto se convierte en un sinfín de tecnicismos que profundizan en estos aspectos y, que como ya hemos señalado, resultan difícilmente comprensibles para las personas no inmersas en el mundo de la Ingeniería Telemática o Informática.

\subsection{García Aretio, L. (2004): "¿Buscar la información o} reproducirla?".

Este tercer documento intenta hacer comprender al lector la importancia que tiene el saber elegir la información en Internet, dada la abundancia de la misma, su constante actualización y el peligro de acceder a muchas carentes de calidad e incluso perjudiciales y poco contrastadas. 
Comenta las ventajas que tiene frente a la documentación impresa en cuanto a rapidez y cantidad se refiere, señalando igualmente las desventajas o inconvenientes que ya hemos reseñado.

Una frase que podría servir como conclusión a este texto podría ser "saber buscar y seleccionar información, aprender a buscarla y seleccionarla o enseñar a otros a adquirir esas destrezas, se convierte hoy en un objetivo imprescindible, sobre todo en un ámbito de enseñanza-aprendizaje".

Una única cuestión quedaría por resolver: quién, cuándo y cómo se llevarán estos objetivos a los Proyectos Curriculares para comenzar su tratamiento desde las más tiernas edades y acostumbrar a nuestros alumnos a trabajar con estas Nuevas Tecnologías. Esperemos que estas dudas tengan una pronta solución.

\subsection{Hsu, S.; Marques, O.; Hamza, M.K. y Alhalata, B. (1999): "How to design a Virtual Classroom: 10 Easy Steps to Follow".}

Antes de comenzar la crítica a los dos siguientes documentos me gustaría reflejar que, si bien el hecho de que estén escritos en inglés puede suponer en algún caso un esfuerzo añadido haciendo más laboriosa su comprensión, debo aclarar que resulta bastante accesible y fácil de entender aun con un simple nivel medio de dicho idioma.

El título nos desvela claramente el contenido que va a tratar el texto: "Cómo planificar una Clase Virtual: 10 fáciles pasos a seguir". Más tarde, en la introducción se nos refiere que para llevar a buen fin cada uno de estos diez pasos, a su vez se realizarán unas preguntas cuya respuesta será la clave para conseguirlo. Veamos los pasos que hay que seguir y las preguntas a las que debemos hallar respuesta: 
- Paso 1: Determinar las necesidades y las condiciones necesarias para su satisfacción.

- ¿Hay estudiantes alejados para este Curso?

- ¿Podrán tener acceso al sitio del curso y realizar todas las interacciones necesarias?

- ¿Hay ayuda e intereses institucionales?

- ¿Hay políticas y procedimientos administrativos para estos casos?

- Paso 2: Estimar el coste, el esfuerzo, y las implicaciones del desarrollo.

- ¿Cuánto tiempo va a tomar desarrollar la clase virtual?

- ¿Qué herramientas serán necesarias? ¿Cuánto costarán? ¿Qué equipo requerirán?

- ¿Cuánta gente y con qué especialidad será necesaria?

- ¿Cuánto afectará el desarrollo en la carga de trabajo del instructor?

- ¿Cuánto afectará a los métodos de enseñanza usados hasta ahora?

- ¿Cuáles son las políticas a seguir para publicar la Web de la institución y el acceso a Internet?

- ¿Cuánto del contenido total está ya disponible en formato electrónico?

- ¿Cuáles serán los requisitos de software/hardware para la máquina del cliente (estudiante)?

- ¿Cuáles serán los requisitos de software/hardware para la máquina del servidor? 
- Paso 3: Planificar la clase virtual.

- ¿Cuáles son los objetivos educativos?

- ¿Cómo serán logrados estos objetivos?

- ¿Qué objetivos posiblemente no serán logrados y cómo podemos compensarlo?

- ¿Qué se podía utilizar para alcanzar un objetivo dado? ¿Cuál de ellos es más viable?

- Paso 4: Diseñar la sala de clase virtual.

- ¿Cómo serán estructuradas las páginas HTML (quizá millares)?

- ¿Podemos proporcionar un "mirar y sentir interés" (preferiblemente constante) " para las páginas Web?

- ¿Cuál debe ser la disposición para una página típica del curso?

- ¿Cómo se supone que deben ser las páginas? ¿Cuál es la relación hardware/software/tecnología?

- $\quad$ Paso 5: Preparar y distribuir el contenido.

- ¿Qué herramientas de editores deben ser utilizadas?

- ¿Cómo importar, crear, editar las imágenes?

- ¿Cómo importar, crear, editar los sonidos?

- ¿Cómo importar, crear, editar gráficos y animaciones?

- ¿Cómo importar, crear, editar vídeos? 
- ¿Dónde estarán las páginas Web mostradas?

- $\quad$ Paso 6: Establecer la comunicación.

- ¿Qué forma de comunicación?

- ¿Qué herramientas deben ser utilizadas?

- Paso 7: Implementación de los métodos online de evaluación de los alumnos.

- ¿Debe la clase virtual contener concursos?

- ¿Cómo hacemos evaluaciones en línea?

- ¿Debemos proporcionar alguna evaluación del estudiante?

- ¿Cómo se ejecutan las pruebas en línea?

- ¿Cómo evitar los engaños?

- ¿Cómo serán calificados y devueltos a los estudiantes las evaluaciones, las pruebas, etc.?

- $\quad$ Paso 8: Implementación de los procedimientos de gestión de la clase virtual.

- ¿QQué tipo de información de la clase es relevante?

- ¿Qué herramientas se pueden utilizar para realizar esas tareas? 
- $\quad$ Paso 9: Instalar el sistema.

- ¿Qué componentes de hardware y software son necesarios?

- ¿Qué es necesario instalar para configurar y proteger el contenido de la clase virtual?

- $\quad$ Paso 10: Mantener y actualizar la clase virtual.

- ¿Cuáles son los medios, el software, y las políticas para preservarlo?

- ¿Qué software necesitamos para mejoras y mantenimiento del hardware?

- ¿Se deben mantener regularmente el contenido y los links del sitio?

Para terminar, únicamente señalar la fecha de publicación del texto estudiado (1999), lo que nos puede inducir a sospechar la caducidad de algunas informaciones, dada la velocidad de cambio en lo que a este campo se refiere.

\subsection{Nielsen, J. et al (2001): "Make it usuable".}

"Hágalo útil" es el título del quinto texto elegido, y de igual manera que el anterior, nos muestra pasos a seguir, en esta ocasión para mejorar nuestro sitio web para que los usuarios se sientan cómodos y no les resulte difícil el acceso a algunas páginas con la consiguiente negativa a volver a visitarla. También nos sugieren que antes de lanzar al mercado nuestro sito web, deberíamos invitar aproximadamente a cinco personas para que prueben nuestro producto mientras son observados por nosotros sin ningún tipo de ayuda, consejo o distracción. Los pasos que sería conveniente seguir con nuestros "probadores del producto" serían los siguientes: 
- Determinar los objetivos y escribir las tareas del usuario.

o Dejar a los usuarios decidir qué productos comprarán, cómo comprarlos, etc.

o Numerar y escribir cada tarea en páginas separadas.

- Determinar el perfil de usuario y programar las sesiones.

o Hay que elegir a personas representativas.

o No tener a todos los usuarios al mismo tiempo y pasar con cada uno entre 20 minutos y dos horas.

o Ofrecer algún incentivo.

- Conducir las sesiones.

o Procurar comodidad a los probadores.

o Resistir la tentación de ayudarlos.

- Evaluar los datos.

o No buscar excusas para los fallos de diseño.

o Cambiar lo que provocara frustración o rechazo en los usuarios.

o Repetir la observación con los cambios pertinentes.

- Después de todo, se puede hacer más.

o Estos grupos de prueba, aunque nos aportan ventajas, tampoco están exentos de problemas, pues son menos exactos informativamente e incluso pueden ser engañosos.

Como valoración general, lo que este documento refleja es bastante sencillo de conseguir y poco costoso, por lo que se constituye en consejos muy prácticos y factibles para cualquiera que pretenda crear un sitio web propio, aunque no obtenga unos niveles óptimos de fiabilidad, pero sin la necesidad de hacer una gran 
investigación ni de precisar de laboratorio, lo que generalmente es inviable para la mayoría de los usuarios.

\subsection{Farías, M. y Ponce, R.M. (2004): "Diseño de páginas web para ambientes virtuales de aprendizaje".}

Práctico documento de gran utilidad para los que pretenden crear una página web educativa para una clase, nos comenta los distintos componentes que debe tener la misma:

- Anuncios o avisos generales.

- Distribución de tareas y calificaciones.

- Recolección de tareas y registro de calificaciones.

- Distribución de notas sobre los temas expuestos por el profesor.

- Exámenes on line.

- Discusiones en grupos y la utilización del correo electrónico.

- Retroalimentación de los estudiantes hacia el profesor.

- Verificación de las calificaciones por parte de los estudiantes.

- También comenta la conveniencia de introducir una bitácora.

El documento estudiado termina añadiendo unos datos estadísticos en cuanto a los resultados obtenidos con grupos de estudiantes utilizando este tipo de páginas web (grupos experimentales) y otros grupos que no tuvieron acceso a ellas (grupos de control), concluyendo que el beneficio para los alumnos en los grupos experimentales fue su mejora académica por la cantidad y calidad de información adquirida, los conceptos científicos utilizados en la resolución de problemas y en la complejidad de la comprensión de la información relevante. 
Resulta un texto muy explícito y motivador que anima a los educadores para hacer uso de estas Nuevas Tecnologías con el fin de conseguir mejores resultados y no quedarse obsoletos en cuanto a recursos, convirtiéndose en piezas de museo.

\section{Análisis de ejemplos de herramientas educativas en Internet.}

Intentaremos ahora analizar la utilidad en contextos educativos de ejemplos concretos de distintas herramientas (Webquest, Weblog, Hotpotatoes, ePortfolio, etc.), señalando en cada una de ellas URL y pantalla del programa, URL y pantalla de ejemplo, y sus ventajas educativas.

Se han seleccionado como recursos a tratar algunas de las que podríamos clasificar como vías de intercomunicación virtual entre los distintos miembros de una Comunidad Educativa. Siendo muchas las posibilidades de elección (weblog, foros, chat, correo, listas de distribución, msn...), nos centraremos en dos de ellas frecuentemente utilizadas en ambientes educativos: el Blog y el Foro.

\subsection{Blog.}

En cuanto al blog (weblog, bitácora) comenzaremos transcribiendo su significado extraído de la Enciclopedia Libre Wikipedia:

"Un weblog, también conocido como blog o bitácora (listado de sucesos), es un sitio web periódicamente actualizado que recopila cronológicamente textos o artículos de uno o varios autores donde el más reciente aparece primero, con un uso o temática en particular, siempre conservando el autor la libertad de dejar publicado lo que crea pertinente. Los weblogs usualmente están escritos con un estilo personal e informal". 
Otras definiciones de weblog: la nueva forma de hacer sitios web dinámicos; diario privado a la vista de todos en Internet; página web hecha de segmentos (de texto o fotos) generalmente cortos y ordenados cronológicamente, similar a una página de resumen de noticias en un periódico; donde uno o más autores publican regularmente sus reflexiones, con enlaces a noticias u otras webs; sitios amateur de noticias y recomendaciones escritas por los internautas.

Para la creación y/o utilización de esta herramienta existen diferentes posibilidades en la web (blogspirit.com, bitacoras.com, blogs.ya.com, yaicos.com...) No obstante, las más accesibles y frecuentemente utilizadas son blogger.com y blogia.com. Siendo sus características muy similares, nos centraremos en el primero de ellos.

2.1.1 URL y pantalla del programa: http://www.blogger.com

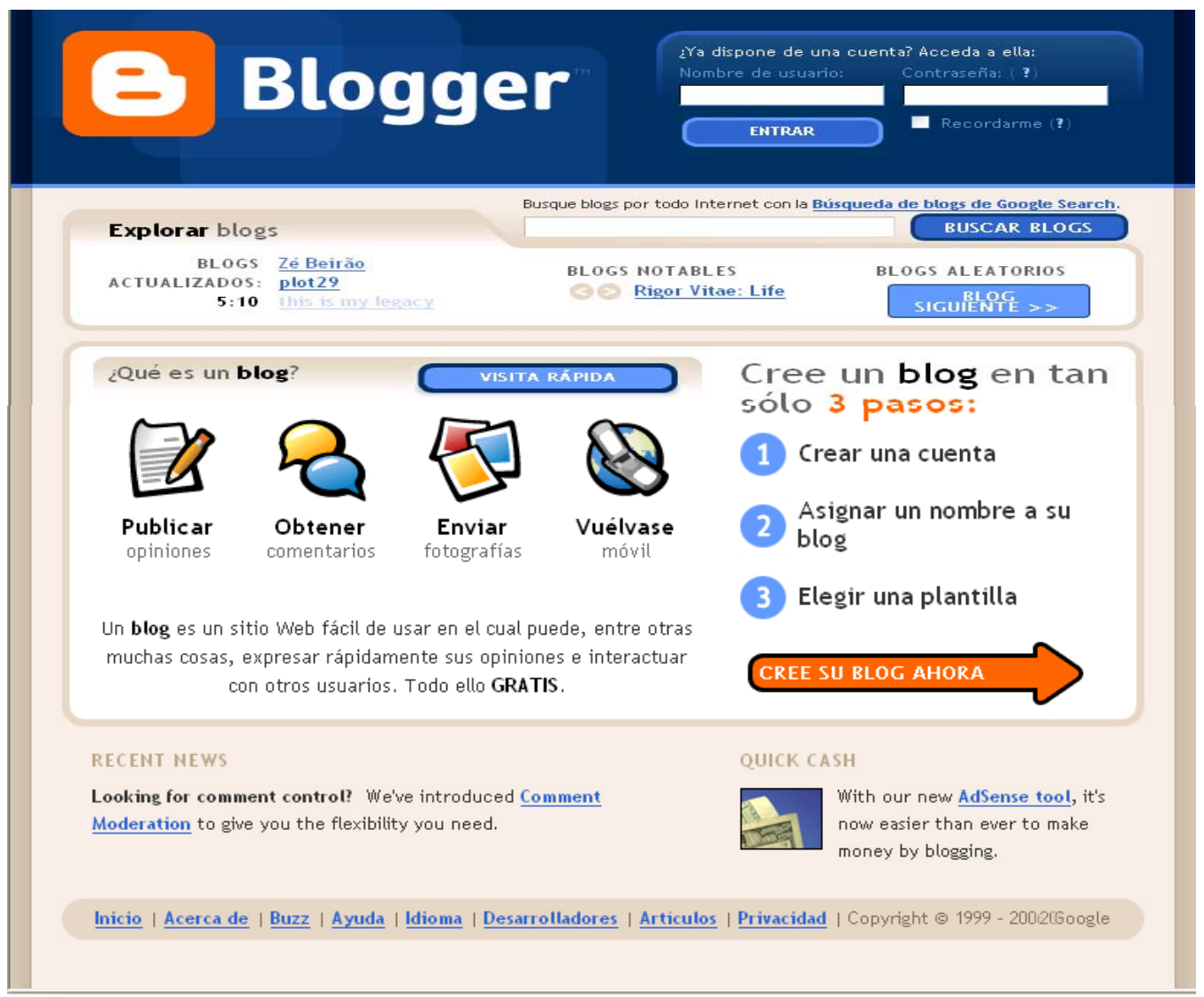




\subsubsection{URL y pantalla de ejemplo: http://paginas-web educativas.blogspot.com}

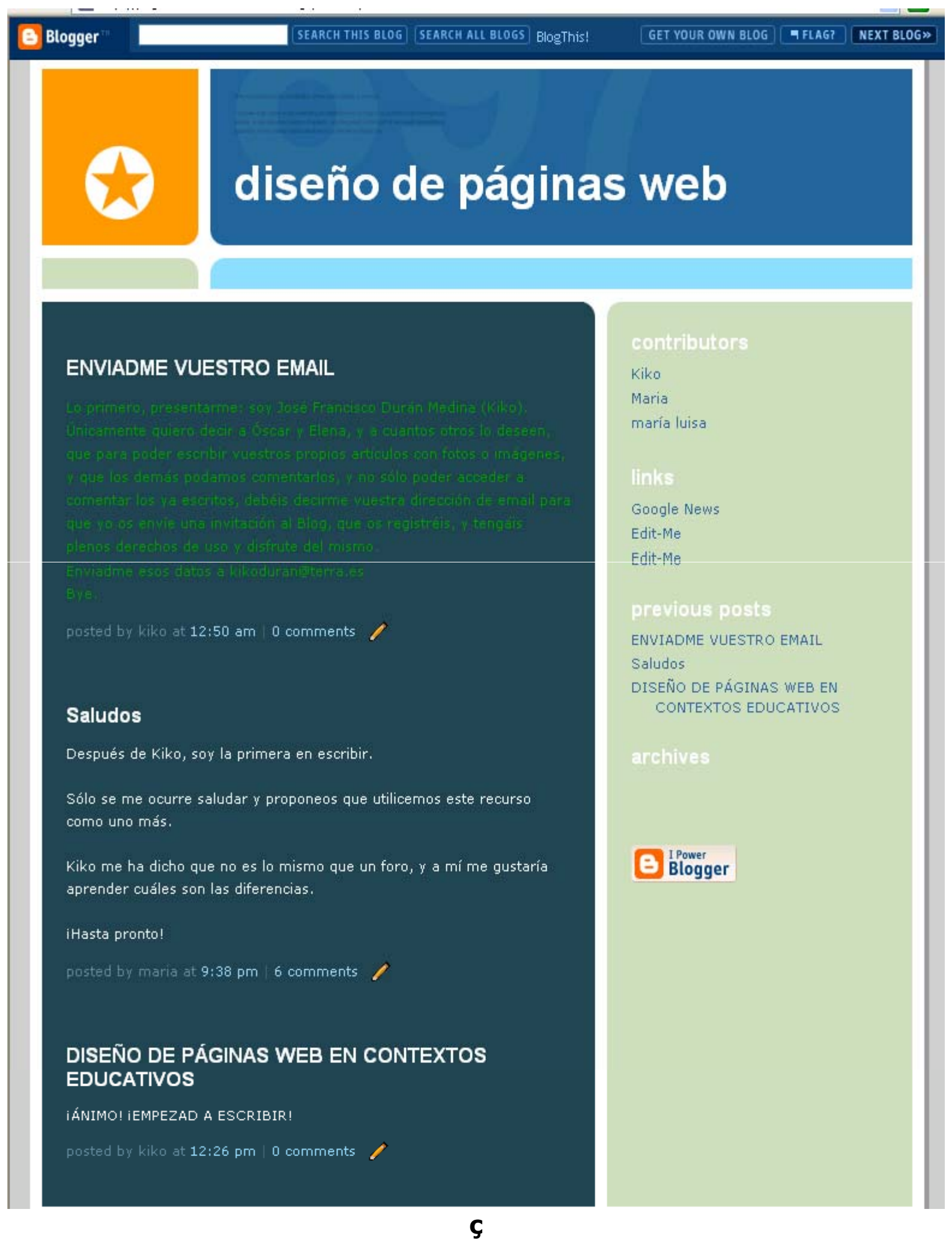




\subsubsection{Ventajas educativas.}

En todos estos ejemplos podemos observar los elementos integrantes de la página que, aunque puedan presentarse en distinta localización o con distinto formato, son los siguientes:

- Título del blog.

- Miembros (Contributors): lista de los participantes registrados en el blog con la posibilidad de acceder a su perfil y a todos los blogs en los que está registrado.

- Escritos recientes (Recent Posts): Distribuidos por títulos de los artículos escritos.

- Archivos (Archives): clasificados por fechas, en este caso por meses de redacción de escritos.

- Entradas (Posts): donde nos indican la fecha, el título, el texto entero, el autor, la hora de realización del escrito, y nos ofrecen la posibilidad de hacer algún comentario a lo leído.

- Y también diversas pestañas con variadas funciones, como la de escribir una entrada (Blog this), búsqueda en este u otros blogs Search...), visita a otros blogs (next blog), creación de un nuevo blog (get your own blog)...

En cuanto a las ventajas educativas que ofrece y ateniéndonos a las experiencias personales que hemos podido obtener, así como a las experiencias ajenas conocidas y comentadas, invitamos a los lectores de este trabajo para que visiten y comprueben por sí mismos en cualquier blog educativo accesible, la extraordinaria motivación e interés que despierta esta herramienta en los alumnos, acrecentando y potenciando entre ellos la cooperación, solidaridad y afán de investigación, amén de otros muchos aspectos educativos como pueden ser la consecución de una correcta forma de escribir textos, el aprendizaje cooperativo, la búsqueda de información fiable, etc. 


\subsection{Foro.}

También podemos encontrar en Internet variadas definiciones de lo que es un Foro:

- Los foros en internet son también conocidos como foros de mensajes o foros de discusión y son una aplicación web que le da soporte a discusiones en línea. Son los descendientes modernos de los sistema de noticias BBS (Bulletin Board System) y Usenet, muy populares en los años 1980 y 1990.

es.wikipedia.org/wiki/Foro_(internet)

- Espacio virtual creado en Internet o en una Intranet en donde los usuarios pueden enviar y contestar mensajes que pueden ser leídos por otros usuarios. www.campusformacion.com/glosario.asp

- Área de una BBS o servicio telemático que versa sobre un tema. Se dejan mensajes a los que puede acceder cualquier persona que visite la conferencia. comunidad.derecho.org/mjviega/publicac/glosario.htm

- Sistema que permite discutir sobre un tema determinado. Los usuarios insertan y leen opiniones, comentarios... Los foros no son en tiempo real y, a no ser que sean específicamente borrados, los mensajes perduran para posteriores consultas. www.portalabogados.com.ar/glosario/informatico.php

- Punto de encuentro en Internet en el que un grupo de personas interesadas en un mismo tema se comunican e intercambian opiniones, preguntas, dudas... www.portal-uralde.com/dicf.htm

De igual manera que ocurre con los blogs, también existen distintas direcciones web que permiten la creación de foros (my-forum.org, latin-foros.com...). Y también en este caso vamos a centrar nuestro estudio en uno de los habitualmente utilizados: superforos.com. 
En cuanto a los foros, comentaremos en la siguiente página la creación de los mismos a través de superforos.com.

\subsubsection{URL y pantalla del programa: http://www.superforos.com}

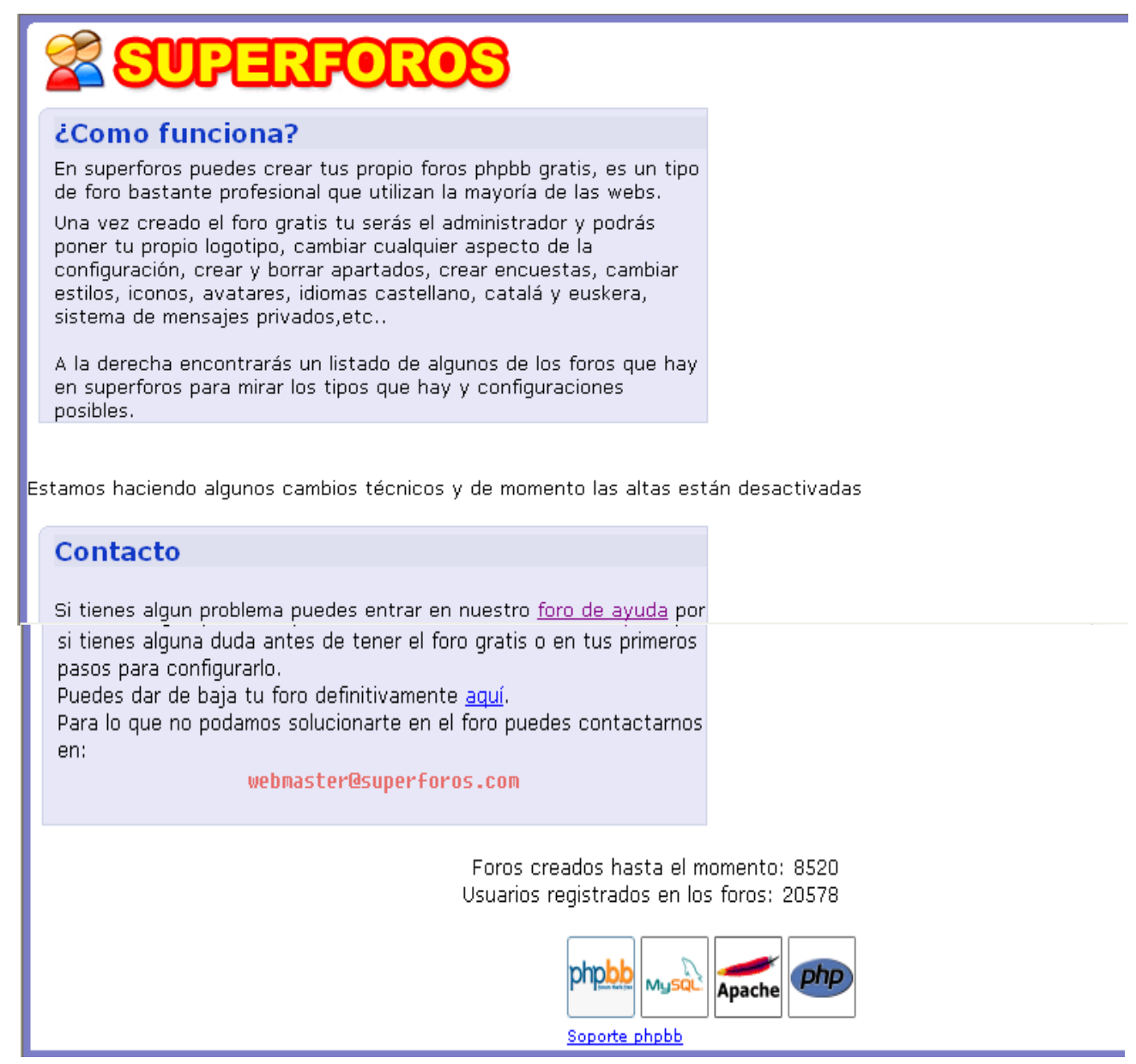

Como podemos observar, se están llevando a cabo algunos cambios técnicos en esta página y de momento las altas están desactivadas, modificando el aspecto general de la misma. Intentaremos estar atentos para poder incorporar la imagen habitual en cuanto esté solventado el problema. No obstante, podemos decir que es bastante similar al del blog visto anteriormente, ofreciendo la posibilidad de crear un foro, 
darle distintos formatos, colores de fondo y letra, títulos, temas dentro de un mismo foro, etc.

2.2.2. URL y pantalla de ejemplo: http://nntt.superforos.com

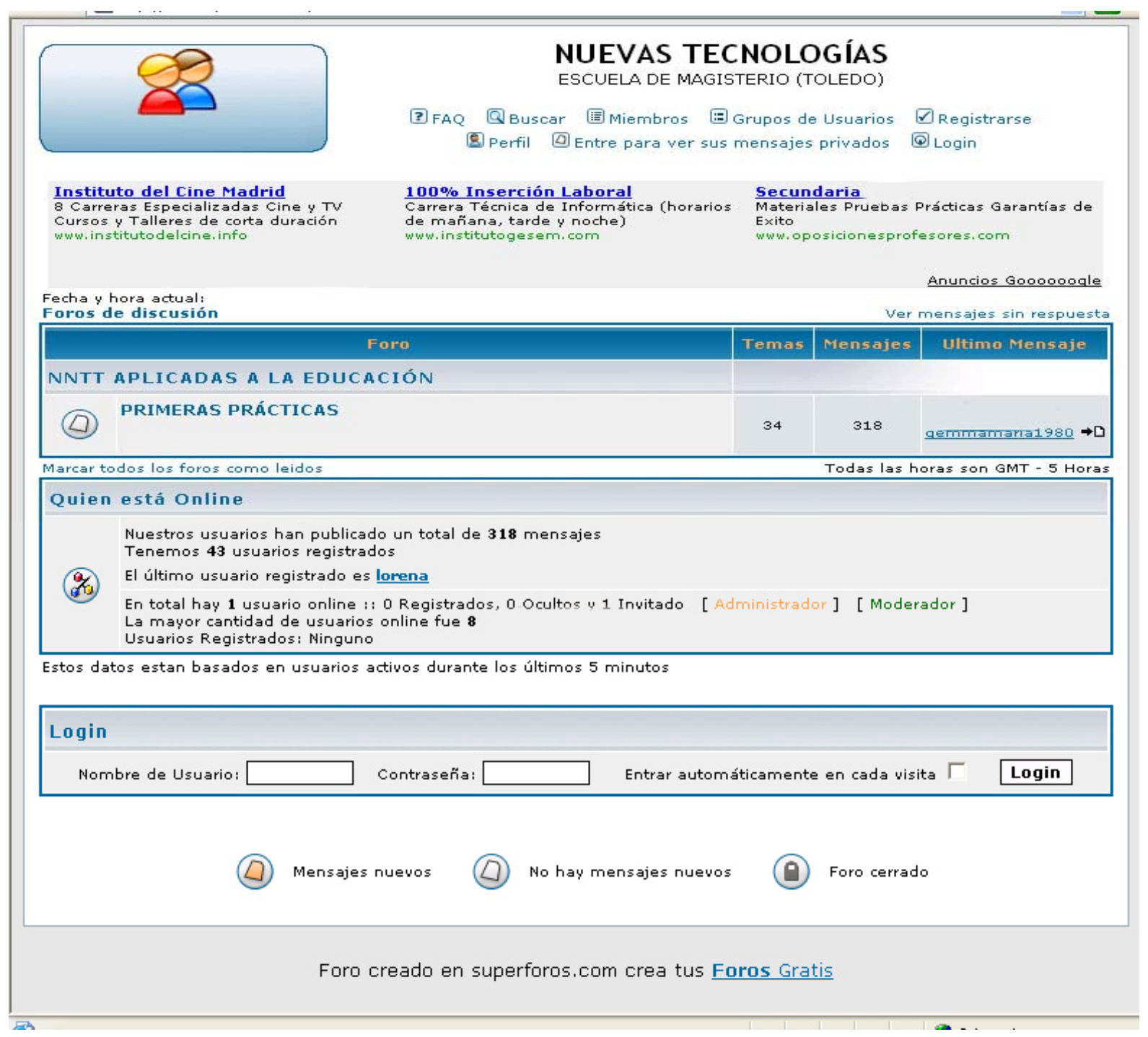

Y accediendo al foro que elijamos, la pantalla que aparece muestra este aspecto: 


\section{PRIMERAS PRÁCTICAS}

(9 nuevotema Foros de discusión - P PRIMERAS PRÁCTICAs

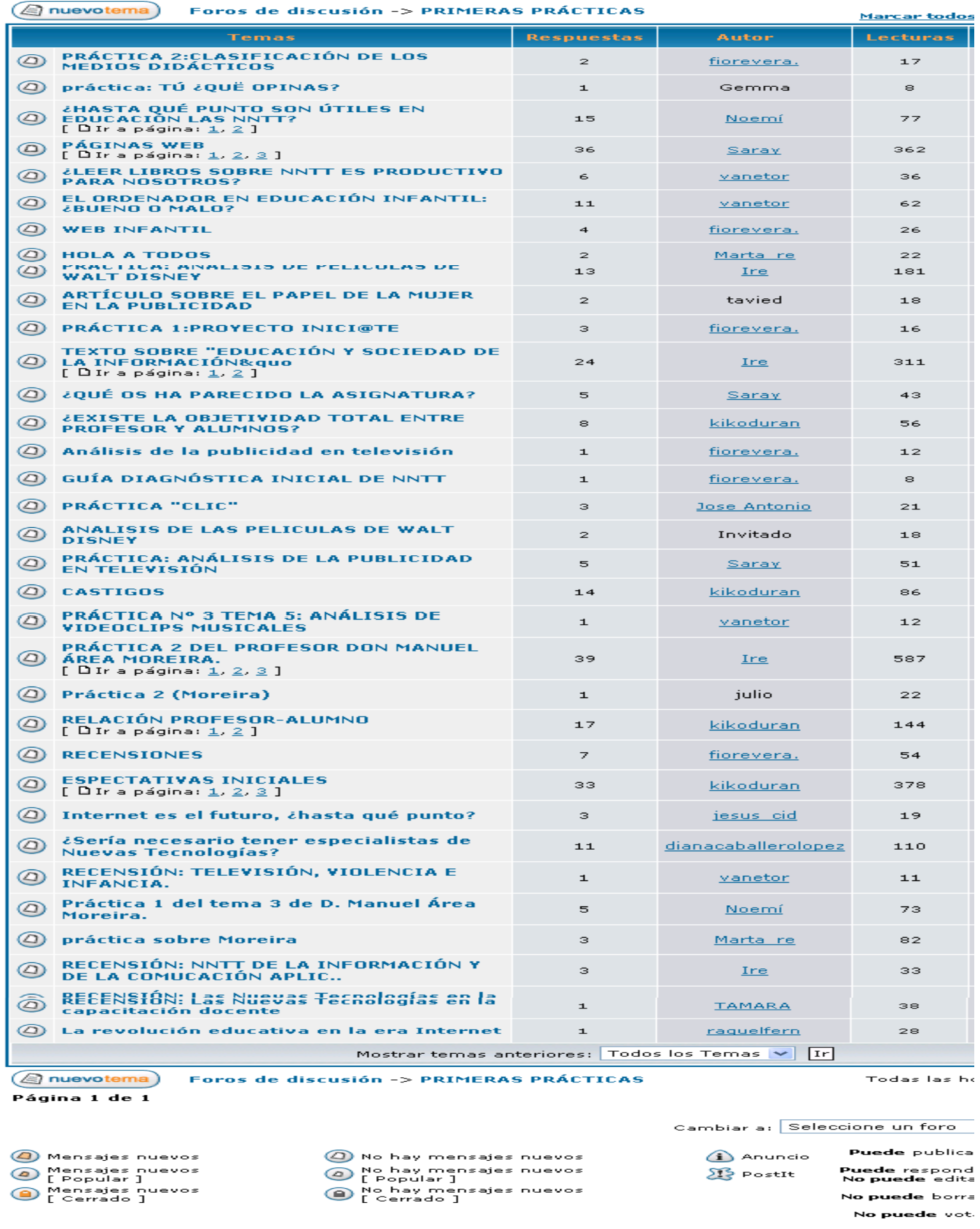




\subsubsection{Ventajas educativas.}

Si queremos hacer un repaso a las funciones que nos ofrece esta web para poder observar mejor sus ventajas educativas, son las siguientes:

- Título del foro.

- Título de los diferentes apartados que componen el foro. En nuestro caso sólo uno (Primeras Prácticas) con información de cada uno de ellos, referente a los temas tratados, los mensajes recibidos y fecha, hora y autor del último.

- Información diversa: usuarios registrados, usuarios en línea, mensajes nuevos...

- De igual manera que ocurría en el blog, diversas pestañas con funciones como preguntas frecuentes (sirven como ayuda), buscar (dentro del foro con palabras clave), miembros (lista con información), grupos, registrarse, perfil, login (para identificarse)...

Y entrando en los apartados señalados, como podemos observar en la segunda pantalla-ejemplo ofrecida, accederíamos a una página donde se desglosan todos los temas tratados y nos informan del autor y creador de ese tema, lecturas que ha tenido, último mensaje, fecha, etc.:

¿Ventajas educativas? Por supuesto. Podríamos decir, sin ánimo de resultar repetitivos que el foro nos aporta las mismas ventajas descritas en el blog: interés, motivación, investigación, cooperación, solidaridad.... Quizás la única diferencia que hemos podido observar ha sido la distinción en cuanto al tratamiento de los temas en uno y en otro. Mientras que el blog es utilizado de una manera más familiar, como el diario de "andar por casa", utilizando un lenguaje más coloquial (sin llegar a ser vulgar), haciendo uso de abreviaturas, cometiendo algunos errores ortográficos, etc., en el foro, sin embargo, es más común una utilización culta del lenguaje, con 
tecnicismos, seriedad y sobriedad, aportando datos y, por lo general, una opinión personal contrastada y documentada.

Si todavía queda alguna duda del aprovechamiento educativo que se puede extraer de esta herramienta, no hay más que observar con detenimiento la pantalla de ejemplo y comprobar la amplitud de su aceptación por parte del alumnado, con 43 usuarios registrados, 34 temas y 318 mensajes.

\section{Conclusiones.}

Podemos concluir que ambas herramientas son bastante aceptadas para llevar a cabo una interrelación comunicativa entre profesor-alumnos y entre alumnoalumnos.

Se puede comprobar la utilidad, gratuidad y simplicidad en el manejo de los blogs y los foros en sus pantallas de presentación, dando múltiples opciones a los seleccionados como administradores, posibilitando modificaciones en el aspecto exterior de los mismos (formato, colores, idioma, presentación...), así como en la apertura hacia el exterior (accesible públicamente, sólo para usuarios registrados, etc.) y suficiente ayuda técnica para poderlos crear en sencillos pasos.

En cuanto a la utilidad educativa creemos suficientemente demostrado con los ejemplos extraídos de la Escuela de Magisterio de Toledo de la Universidad de Castilla La Mancha, la gran motivación e interés que despierta en los alumnos provocando una amplia utilización, tanto en el número de miembros participantes como en el número de mensajes escritos.

Terminamos este apartado señalando algo anteriormente comentado: la diferencia entre estas herramientas en cuanto a la redacción de los escritos y la relación mantenida por los usuarios, quizás debido más a la acción del administrador que al 
propio recurso utilizado, otorgando generalmente al blog un tratamiento y aspecto más informal que a los foros.

\section{Bibliografía.}

ADELL, J. (1996). "Internet en Educación: una gran oportunidad" en Revista Net Conexión, no 11. Septiembre 1996. Disponible en:

http://tecnologiaedu.us.es/cuestionario/bibliovir/19.pdf (Consultado el 02/01/2005).

FARÍAS, M. y PONCE, R.M. (2004). "Diseño de páginas web para ambientes virtuales de aprendizaje"; en Revista RMIE-Revista Mexicana de Investigación Educativa, no 20. Enero -Marzo 2004. Disponible en http://dialnet.unirioja.es

GARCÍA, L. (2004). "¿Buscar la información o reproducirla?" en Revista BENED, Editorial. Mayo 2004. Disponible en: http://www.uned.es/bened (Consultado el 02/01/2005).

HSU, S.; MARQUES, O.; HAMZA, M.K. y ALHALATA, B. (1999). "How to design a Virtual Classroom: 10 Easy Steps to Follow" en Revista ERIC, n० ED437027. Disponible en http://www.eric.ed.gov (Consultado el 02/01/2005).

MARQUÉS, P. (1999). "Concepciones sobre el aprendizaje" en Revista DIM. 1999. Disponible en: http://dewey.uab.es/pmarques (Consultado el 02/01/2005).

NIELSEN, J.; COYNE, K. y TAHIR, M. (2001). "Make it usuable" en Revista PCMAG. Febrero 2001. Disponible en http://www.pcmag.com/article2/0,4149,33821,00.asp (Consultado el 02/01/2005).

SANTACRUZ-VALENCIA, L.P., AEDO, I. y DELGADO-KLOOS, C. (2004). "Objetos de aprendizaje: tendencias dentro de la web semántica"; en Boletín de RedIRIS, no 6667. Diciembre 2003 - enero 2004, pp. 76-79. Disponible en: 
http://www.rediris.es/rediris/boletin/66-67/ponencia18.pdf (Consultado el 02/01/2005).

\section{Webgrafía}

comunidad.derecho.org

nntt.superforos.com

www.bitacoras.com

www.blogs.ya.com

www.blogspirit.com

www.campusformacion.com

www.latin-foros.com

www.my-forum.org

www.portalabogados.com

www.portal-uralde.com

www.superforos.com

www.yaicos.com

www.wikipedia.org 